\section{Making everyday clinical life easier}

Directa Dental Group's products are developed and evaluated by a team of highly-qualified and renowned Swedish and international dental professionals with the aim of making life easier for dental professionals in daily clinical work. Product categories include ProphyCare (pastes and cups), PractiPal (colourful trays, storage and stands), luxators, DryDent for saliva and moisture control, restorative tools, root irrigators and much more!

Directa Dental Group is highly proactive on the international dental market with business partnerships in many parts of the world. With headquarters in Sweden, offices, manufacturing, and distribution and more than 200 employees representing the various brands worldwide, Directa Dental Group is an innovative player in the global dental arena. The company attends and exhibits at most major international dental events every year, and works incessantly in creating new and successful partnerships all over the world.

Directa Dental Group currently consists of Directa $A B$, Orsing $A B$, Topdental (Products) Ltd and Parkell, Inc. The companies are all managed independently, having full responsibility and autonomy for their own operations.

For more information on Directa dental products visit www.directadental.com.

\title{
An incredibly versatile adhesive on display
}

GC will be exhibiting all their latest innovations as well as all their tried and trusted favourites on stand $\mathrm{H} 10$ at this week's BDIA Dental Showcase.

Of particular interest will be GC's NEW G-Premio BOND, which is incredibly versatile because it is compatible with all total-etch, self-etch and selective etch techniques.

HEMA free, G-Premio BOND is a one component, universal bonding agent that is perfectly adapted to all direct restorations and can also be used to repair indirect restorations without the need for a primer. It is equally suitable for use in combination with a silane when repairing glass or hybrid ceramics, and is ideal as a treatment for hypersensitivity.

G-Cem LinkForce is the latest addition to GC's comprehensive luting portfolio. Building on the success of the G-Cem brand, G-Cem LinkForce is their NEW dual cure

universal luting cement.
It is a universal adhesive resin cement that ensures a strong bond to all substrates - in all indications, without compromises.

Available in four shades, accompanied by try-in pastes, and tooth-like fluorescence, G-Cem LinkForce combines high bond strength with ease of use, stress-free clean-up of any excess, superb colour stability, very low $3 \mu \mathrm{m}$ film thickness and superior wear resistance. It also offers dual-curing convenience - efficient self-curing, particularly useful when luting opaque or thick restorations, and optimal light-curing, ideal for veneers.

There is also the opportunity to see EQUIA Forte, the latest innovation in GC's glass ionomer and resin technology range, and Essentia, their new light-cured radiopaque universal composite range.

For further information contact GC UK Ltd on 01908218999 or visit www.gceurope.com.

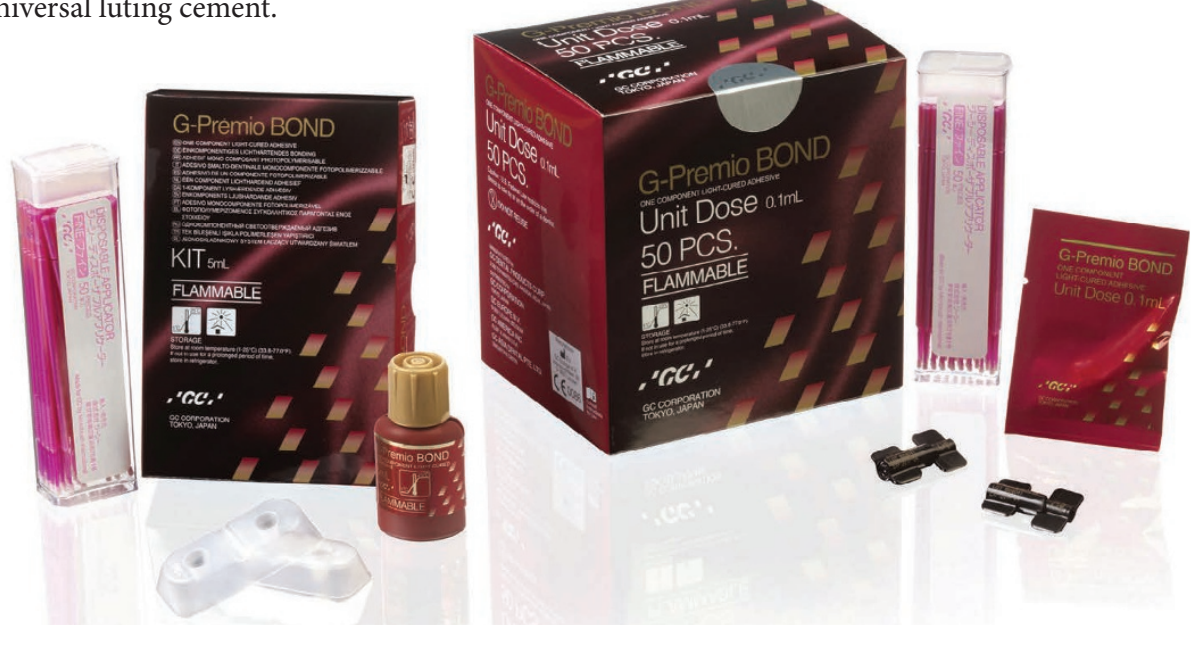

\section{Comprehensive occlusion and bite splint course}

Mizrahi Dental Teaching is offering a new, unique and comprehensive practical occlusion 'hands-on' course over three days presented by Dr Basil Mizrahi.

Rather than gloss over various confusing and complicated concepts and philosophies, it will focus on the nuts and bolts of occlusion such as occlusal contacts and tooth morphology and build up to more complex aspects such as occlusal adjustment.

Most of the course will be made up of practical exercises and use large models and drawings which make learning much easier. Delegates will also learn about the difference between various types of bite splints and which one to use when. Each delegate will have the opportunity to fit and adjust an upper 'Michigan' bite splint.

The aim of the course is to demystify and simplify occlusion so delegates can come away feeling less intimidated by occlusion. Delegates will understand when occlusion is important and when it's not and be able to implement what they have learnt into their clinical practice.

Topics covered will include:

- How to make lucier jigs and take a facebow and bite registration

- Facebows - different types and their limitations. Why even use one?

- Bite registration records - different techniques and their pros and cons. When do you need to use them?

- What do posterior teeth look like, what cusp fits into what fossa and why. How to assess and adjust high spots when fitting and crowns and bridges

- How to shape temporary crowns and posterior composites

- Splints - which ones to use, why and when?

- How to fit and adjust an occlusal splint

- How to adjust an occlusion.

For more information on this and other upcoming courses visit www.mizrahi-dental-teaching.co.uk. 